VOL. $66(2002)$ [443-463]

\title{
LOCAL WELL POSEDNESS FOR STRONGLY DAMPED WAVE EQUATIONS WITH CRITICAL NONLINEARITIES
}

\author{
Alexandre N. Carvalho and Jan W. Cholewa
}

In this article the strongly damped wave equation is considered and a local well posedness result is obtained in the product space $H_{0}^{1}(\Omega) \times L^{2}(\Omega)$. The space of initial conditions is chosen according to the energy functional, whereas the approach used in this article is based on the theory of analytic semigroups as well as interpolation and extrapolation spaces. This functional analytic framework allows local existence results to be proved in the case of critically growing nonlinearities, which improves the existing results.

\section{INTRODUCTION}

For $\theta \in[1 / 2,1], \eta>0$, consider the family of problems

$$
\left\{\begin{array}{l}
u_{t t}+\eta(-\Delta)^{\theta} u_{t}+(-\Delta) u=f\left(u, u_{t}\right), t>0, x \in \Omega, \\
u(0, x)=u_{0}(x), u_{t}(0, x)=v_{0}(x), x \in \Omega \\
u(t, x)=0, t \geqslant 0, x \in \partial \Omega
\end{array}\right.
$$

where $\Omega$ is a bounded smooth domain in $\mathbb{R}^{n}, n \geqslant 3$, and $A=(-\Delta)$ with Dirichlet boundary conditions. It is well known that $A$ is a positive, self-adjoint operator with domain $D(A)=H^{2}(\Omega) \cap H_{0}^{1}(\Omega)$ and therefore $-A$ generates a compact analytic semigroup on $X=X^{0}=L^{2}(\Omega)$. Denote by $X^{\alpha}$ the fractional power spaces associated with the operator $A$; that is, $X^{\alpha}=D\left(A^{\alpha}\right)$ endowed with the graph norm.

The problems (1) will be viewed as ordinary differential equations in a product space $Y=Y^{0}=X^{1 / 2} \times X^{0}$ :

$$
\frac{d}{d t}\left[\begin{array}{l}
u \\
v
\end{array}\right]+\mathcal{A}_{(\theta)}\left[\begin{array}{l}
u \\
v
\end{array}\right]=\mathcal{F}\left(\left[\begin{array}{l}
u \\
v
\end{array}\right]\right), t>0,\left[\begin{array}{l}
u \\
v
\end{array}\right]_{t=0}=\left[\begin{array}{l}
u_{0} \\
v_{0}
\end{array}\right],
$$

\section{Received 2nd april, 2002}

This work was carried out while the second author visited Instituto de Ciências Matemáticas e de Computação at Universidade de São Paulo in São Carlos, Brazil. He wishes to acknowledge the hospitality of the people from this Institution.

The first author's research was partially supported by CNPq grant \# 300.889/92-5 and FAPESP grant \# 97/011323-0, Brazil. The second author's research was partially supported by FAPESP grant \# 99/03116-0, Brazil; also by Polish State Committee for Scientific Research (KBN) grant \# 2 PO3A 035 18.

Copyright Clearance Centre, Inc. Serial-fee code: 0004-9727/02 \$A2.00+0.00. 
with $\mathcal{A}_{(\theta)}$ and $\mathcal{F}$ denoted in a matrix form by

$$
\mathcal{A}_{(\theta)}=\left[\begin{array}{cc}
0 & -I \\
A & \eta A^{\theta}
\end{array}\right], \mathcal{F}=\left[\begin{array}{l}
0 \\
F
\end{array}\right]
$$

where $F$ is the Nemitskiu map associated with $f\left(u, u_{t}\right)$, and $\mathcal{A}_{(\theta)}: D\left(\mathcal{A}_{(\theta)}\right) \subset Y^{0} \rightarrow Y^{0}$ with

$$
D\left(\mathcal{A}_{(\theta)}\right)=Y_{(\theta)}^{1}=\left\{\left[\begin{array}{l}
\varphi \\
\psi
\end{array}\right] \in X^{3 / 2-\theta} \times X^{1 / 2} ; A^{1-\theta} \varphi+\eta \psi \in X^{\theta}\right\}, \quad \theta \in\left[\frac{1}{2}, 1\right]
$$

and

$$
\mathcal{A}_{(\theta)}\left[\begin{array}{l}
\varphi \\
\psi
\end{array}\right]=\left[\begin{array}{c}
-\psi \\
A^{\theta}\left(A^{1-\theta} \varphi+\eta \psi\right)
\end{array}\right] \text { for }\left[\begin{array}{l}
\varphi \\
\psi
\end{array}\right] \in D\left(\mathcal{A}_{(\theta)}\right)
$$

We note that the abstract results that we obtain for (2) will cover a wide class of problems that contains, besides (1), some higher order equations. In the applications we shall however focus on the particular example (1).

The homogeneous problem (2) corresponds to the following second order ordinary differential equation in the Hilbert space $Y^{0}$ :

$$
\ddot{u}+\eta A^{\theta} \dot{u}+A u=0, t>0, u(0)=u_{0}, \dot{u}(0)=v_{0} .
$$

This equation has been extensively studied by Chen and Triggiani in a series of papers $[4,5,6]$. In $[4,5]$ the authors prove the sectoriality of the operators $\mathcal{A}_{\theta}, \theta \in[1 / 2,1]$ and discuss some of its spectral properties. In [6], the authors describe fractional power spaces $Y^{\alpha}, \alpha \in[0,1]$, associated with $\mathcal{A}_{\theta}, \theta \in[1 / 2,1]$. Many of our considerations will be based on the results proved in these papers.

We choose as a base space for (1) the product space $Y^{0}=X^{1 / 2} \times X^{0}$. This choice of space seems to be the appropriate choice to study the asymptotic behaviour of (1), for in it we may exhibit an energy functional to (1) (we shall exploit the asymptotic behaviour of (1) in another publication). This choice also allow us to take advantage of the results obtained in $[\mathbf{4}, \mathbf{5}, \mathbf{6}]$.

As we shall see, the structure of the fractional power scale $\left[Y^{\alpha}, \alpha \in R^{+}\right]$as well as the description of the extrapolated fractional power scale generated by $\left(Y^{0}, \mathcal{A}_{(\theta)}\right)$ (see $[1]$ for a definition) is more complicated in the case $\theta \in(1 / 2,1]$ than in the case $\theta=1 / 2$. However for $\theta \in(1 / 2,1]$, we are still able to describe a part of the extrapolated scale needed to solve (1) with $f$ growing with respect to $u$ up to the critical exponent $(n+2) /(n-2)$.

An important remark, at this point, is that the critical exponent for $f$ with respect to $u$ does not depend on the exponent $\theta$ of the damping operator.

For the case $\theta=1$ we shall see that a map $f$ growing like $|u|^{(n+2) /(n-2)}$ is subcritical in the sense of [2]. However it does not seem possible to obtain a local well posedness 
result for maps growing faster than that. This is related to the special smoothing shown in the characterisation of the fractional power spaces associated with $\mathcal{A}_{(1)}$ (see [6]).

It is worth mentioning the admissible growth of the nonlinearity with respect to $u_{t}$. Contrary to the case $\theta=1 / 2$, growth like $\left|u_{t}\right|^{(n+2) /(n)}$ becomes subcritical if $\theta \neq 1 / 2$. We shall see that even faster growth is possible here. The nonlinearity in (1) will be allowed to grow as fast as $\left|u_{t}\right|^{(n+4 \theta) /(n)}$. The criticality of this nonlinearity resembles (it is not exactly the same) the ultra-critical nonlinearities in the terminology of [2].

The main result in this paper concerning local existence is

THEOREM 1. If $f$ satisfies

$$
\begin{aligned}
\left|f(u, v)-f\left(u^{\prime}, v^{\prime}\right)\right| \leqslant c\left|u-u^{\prime}\right|(1 & \left.+|u|^{\rho_{1}-1}+\left|u^{\prime}\right|^{\rho_{1}-1}\right) \\
& +c\left|v-v^{\prime}\right|\left(1+|v|^{\rho_{2}-1}+\left|v^{\prime}\right|^{\rho_{2}-1}\right)
\end{aligned}
$$

with $\rho_{1} \leqslant((n+2) /(n-2))$ and $\rho_{2} \leqslant(n+4 \theta) / n$ then (1) is locally well posed in $H_{0}^{1}(\Omega)$ $\times L^{2}(\Omega)$.

Our aim is to give a functional analytic framework that allow us to exploit the sectoriality of the operators $\mathcal{A}_{(\theta)}$ to obtain sharp local well posedness results for (1). So far, the existing local well posedness results for (1) do not exploit the sectoriality of the operators $\mathcal{A}_{(\theta)}$, and therefore no sharp result is available. We obtain local existence results for critically growing nonlinearities, improving considerably the existing results. This abstract framework is also suitable for the study of the long time behaviour of the semigroup of global solutions to (1) in $Y^{0}$. Results concerning the existence of a global attractor for (1) will be proved in a publication complementary to this.

We remark that we do not assume any kind of sign condition on the map $f$. These sign conditions are usually needed when applying a method like Faedo-Galerkin. We also remark that the $\varepsilon$-regular solutions are more general than the solutions found in [7] in the sense that they do not need to have a controlled growth at the initial time. We use the ideas developed in [2], for it provides a clear framework for dealing with perturbations of linear equations (semilinear equations), extracting the local well posedness results from the knowledge of the fractional power spaces and the properties of the Nemitskii operators associated with the nonlinearity acting on them.

\section{The Strongly Damped Wave Operator}

In this section we study the abstract properties of the operator

$$
\mathcal{A}_{(\theta)}=\left[\begin{array}{cc}
0 & -I \\
A & \eta A^{\theta}
\end{array}\right]: D\left(\mathcal{A}_{(\theta)}\right) \subset X^{1 / 2} \times X \rightarrow X^{1 / 2} \times X
$$

defined by

$$
\mathcal{A}_{(\theta)}\left[\begin{array}{l}
\varphi \\
\psi
\end{array}\right]=\left[\begin{array}{c}
-\psi \\
A^{\theta}\left(A^{1-\theta} \varphi+\eta \psi\right)
\end{array}\right]
$$


for

$$
\left[\begin{array}{l}
\varphi \\
\psi
\end{array}\right] \in D\left(\mathcal{A}_{(\theta)}\right)=\left\{\left[\begin{array}{l}
\varphi \\
\psi
\end{array}\right] \in X^{(3 / 2)-\theta} \times X^{1 / 2} ; A^{1-\theta} \varphi+\eta \psi \in X^{\theta}\right\}
$$

where $A$ is a self adjoint and positive operator in the Hilbert Space $X$, and $X^{\alpha}$ denotes the fractional power spaces associated to $A$.

We remark that

$$
\mathcal{A}_{(\theta)}\left[\begin{array}{l}
\varphi \\
\psi
\end{array}\right]=\left[\begin{array}{c}
-\psi \\
A \varphi+\eta A^{\theta} \psi
\end{array}\right] \text { for }\left[\begin{array}{l}
\varphi \\
\psi
\end{array}\right] \in X^{1} \times X^{\theta}
$$

and that $X^{1} \times X^{\theta}$ is a dense subset of $D\left(\mathcal{A}_{(\theta)}\right)$. However the operator $\mathcal{B}: D(\mathcal{B}) \subset$ $X^{1 / 2} \times X \rightarrow X^{1 / 2} \times X$ defined by $D(\mathcal{B})=X^{1} \times X^{\theta}$ and

$$
\mathcal{B}_{(\theta)}\left[\begin{array}{c}
\varphi \\
\psi
\end{array}\right]=\left[\begin{array}{cc}
0 & -I \\
A & \eta A^{\theta}
\end{array}\right]\left[\begin{array}{l}
\varphi \\
\psi
\end{array}\right]=\left[\begin{array}{c}
-\psi \\
A \varphi+\eta A^{\theta} \psi
\end{array}\right]
$$

is not a closed operator unless $\theta=1 / 2$.

The presentation here will follow closely a series of papers published by Chen and Triggiani in 1988, 1989 and 1990. It was proved in $[4,5]$ that the $\mathcal{A}_{(\theta)}$ is a sectorial operator and therefore it generates an analytic semigroup. Shortly after that, in [6], the authors gave a characterisation of the fractional power spaces associated with $\mathcal{A}_{(\theta)}$, $\theta \in[1 / 2,1]$.

As shown in [2], the knowledge of the scale of fractional power spaces and its embeddings into known spaces are the main tool in obtaining local well posedness of a semilinear sectorial problem (even in the critical growth case). In this sense, this section will provide the main working tools to obtain a general local well posedness result for (1).

Our aim in this section is thus to discuss the properties of $\mathcal{A}_{(\theta)}, \theta \in[1 / 2,1]$, necessary to consider (2) as a sectorial problem on an extrapolation space $Y_{(\theta)_{-1}}$ of $Y^{0}$ generated by $\mathcal{A}_{(\theta)}, \theta \in[1 / 2,1]$.

2.1. Generalities about the operator $\mathcal{A}_{(\theta)}$. In this subsection we establish the basic properties of the operator $\mathcal{A}_{(\theta)}$ that will be used in the main body of the paper. For the sake of completeness we shall sketch the proof of these results. We also remark that in Section 2.1 only Lemma 1 and Corollary 1 make use of the fact that $A$ is a negative Laplacian in $L^{2}(\Omega)$ with $D(A)=H^{2}(\Omega) \times H_{0}^{1}(\Omega)$. All other results are based on the assumption that $A$ is a positive definite, self-adjoint operator in a Hilbert space $X^{0}$ having compact resolvent.

Proposition 1. For each $\theta \in[1 / 2,1]$ the following conditions hold:

(i) $\mathcal{A}_{(\theta)}$ is closed,

(ii) $\mathcal{A}_{(\theta)}$ is maximal accretive or, equivalently, $-\mathcal{A}_{(\theta)}$ is maximal dissipative, 
(iii) $0 \in \rho\left(\mathcal{A}_{(\theta)}\right)$,

(iv) $\mathcal{A}_{(\theta)}$ has compact resolvent provided that $\theta \neq 1$.

(v) $\mathcal{A}_{(\theta)}^{*}=\left[\begin{array}{cc}0 & I \\ -A & \eta A^{\theta}\end{array}\right]$ is the adjoint of $\mathcal{A}_{(\theta)}$ and $D\left(\mathcal{A}_{(\theta)}^{*}\right)=\left\{\left[\begin{array}{l}\varphi \\ \psi\end{array}\right] \in X^{(3 / 2)-\theta} \times X^{1 / 2} ;-A^{1-\theta} \varphi+\eta \psi \in X^{\theta}\right\}$.

(vi) $-\mathcal{A}_{(\theta)}$ generates on $Y^{0}$ a $C^{0}$-semigroup of contractions $e^{-\mathcal{A}_{(\theta)} t}: Y^{0} \rightarrow Y^{0}$, $t \geqslant 0$. Furthermore, the imaginary powers of $\mathcal{A}_{(\theta)}$ are bounded and

$$
\left\|\mathcal{A}_{(\theta)}^{i t}\right\|_{\mathcal{L}\left(Y^{0}, Y^{0}\right)} \leqslant e^{(\pi / 2)|t|}, t \in \mathbb{R}
$$

(vii) $\mathcal{A}_{(\theta)}$ is sectorial operator in $Y^{0}$ with $\operatorname{Re} \sigma\left(\mathcal{A}_{(\theta)}\right)>0$. The semigroup of contractions $\left\{e^{-\mathcal{A}_{(\theta)} t}, t \geqslant 0\right\}$ is thus analytic. It is also compact except for $\theta=1$.

Proof: Note that (i) is immediate from the closedness of $A$ and $A^{\theta}$. For (ii) we first note that

$$
\begin{aligned}
\left\langle\mathcal{A}_{(\theta)}\left[\begin{array}{l}
u \\
v
\end{array}\right],\left[\begin{array}{l}
u \\
v
\end{array}\right]\right\rangle_{Y^{0}} & =\left\langle\left[\begin{array}{c}
-v \\
A^{\theta}\left(A^{1-\theta} u+\eta v\right)
\end{array}\right],\left[\begin{array}{l}
u \\
v
\end{array}\right]\right\rangle_{Y^{0}} \\
& =-\left\langle A^{1 / 2} v, A^{1 / 2} u\right\rangle_{X}+\left\langle A^{\theta}\left(A^{1-\theta} u+\eta v\right), v\right\rangle_{X} \\
& =-\left\langle A^{1 / 2} v, A^{1 / 2} u\right\rangle_{X}+\overline{\left\langle A^{1 / 2} v, A^{1 / 2} u\right\rangle_{X}}+\eta\left\langle A^{\theta / 2} v, A^{\theta / 2} v\right\rangle_{X}
\end{aligned}
$$

Therefore,

$$
\operatorname{Re}\left\langle\mathcal{A}_{(\theta)}\left[\begin{array}{l}
u \\
v
\end{array}\right],\left[\begin{array}{l}
u \\
v
\end{array}\right]\right\rangle_{Y^{0}}=\eta\left\langle A^{\theta / 2} v, A^{\theta / 2} v\right\rangle_{X} \geqslant 0,\left[\begin{array}{l}
u \\
v
\end{array}\right] \in Y_{(\theta)}^{1},
$$

which proves accretivity of $\mathcal{A}_{(\theta)}$. To complete part (ii) it suffices to note that the equation

$$
\left(\mathcal{I}+\mathcal{A}_{(\theta)}\right)\left[\begin{array}{l}
u \\
v
\end{array}\right]=\left[\begin{array}{l}
\tilde{u} \\
\tilde{v}
\end{array}\right]
$$

possesses, for each $\left[\begin{array}{c}\tilde{u} \\ \widetilde{v}\end{array}\right] \in Y_{0}$, a unique solution

$$
\left[\begin{array}{l}
u \\
v
\end{array}\right]=\left[\begin{array}{c}
\left(1+\eta A^{\theta}\right)\left(1+\eta A^{\theta}+A\right)^{-1} \widetilde{u}+\left(1+\eta A^{\theta}+A\right)^{-1} \widetilde{v} \\
-A\left(1+\eta A^{\theta}+A\right)^{-1} \widetilde{u}+\left(1+\eta A^{\theta}+A\right)^{-1} \widetilde{v}
\end{array}\right] \in Y_{(\theta)}^{1} .
$$

Part (iii) is an immediate consequence of the form of the inverse operator

$$
\mathcal{A}_{(\theta)}^{-1}=\left[\begin{array}{cc}
\eta A^{-(1-\theta)} & A^{-1} \\
-I & 0
\end{array}\right] \text {. }
$$


Part (iv) follows from (iii) and compactness of the Sobolev inclusions between $X^{\alpha}$ spaces resulting from compactness of the resolvent of $A$. We remark further that in this paper we are not going to use explicitly the formulas in part $(v)$ concerning the adjoint operator. However, the knowledge of the dual scale $\left(Y^{0}, \mathcal{A}_{(\theta)}^{*}\right)$ may be of some importance in describing the whole two-sided fractional power scale generated by $\left(Y^{0}, \mathcal{A}_{(\theta)}\right)$ (see $[\mathbf{1}$, Chapter V]). To prove part (v), note first that by the density of the embedding $Y_{(\theta)}^{1} \subset Y^{0}$ the adjoint operator $\mathcal{A}_{(\theta)}^{*}$ exists, and since

$$
\left\langle\mathcal{A}_{(\theta)}\left[\begin{array}{l}
u \\
v
\end{array}\right],\left[\begin{array}{l}
\tilde{u} \\
\tilde{v}
\end{array}\right]\right\rangle_{Y^{0}}=\left\langle\left[\begin{array}{l}
u \\
v
\end{array}\right],\left[\begin{array}{cc}
0 & I \\
-A & \eta A^{\theta}
\end{array}\right]\left[\begin{array}{l}
\tilde{u} \\
\tilde{v}
\end{array}\right]\right\rangle_{Y^{0}},\left[\begin{array}{l}
u \\
v
\end{array}\right],\left[\begin{array}{l}
\tilde{u} \\
\widetilde{v}
\end{array}\right] \in X^{1} \times X_{\text {dense }}^{c} Y_{(\theta)}^{1},
$$

we obtain

$$
\mathcal{A}_{(\theta)}^{*}\left[\begin{array}{c}
\tilde{u} \\
\tilde{v}
\end{array}\right]=\left[\begin{array}{cc}
0 & I \\
-A & \eta A^{\theta}
\end{array}\right]\left[\begin{array}{c}
\tilde{u} \\
\tilde{v}
\end{array}\right] \text { for }\left[\begin{array}{c}
\tilde{u} \\
\tilde{v}
\end{array}\right] \in X^{1} \times X^{\theta}
$$

Furthermore,

$$
\begin{aligned}
\mathcal{J}_{(\theta)} & =\left[\begin{array}{cc}
0 & I \\
-A & \eta A^{\theta}
\end{array}\right]: D\left(\mathcal{J}_{(\theta)}\right) \\
& =\left\{\left[\begin{array}{l}
\varphi \\
\psi
\end{array}\right] \in X^{(3 / 2)-\theta} \times X^{1 / 2} ;-A^{1-\theta} \varphi+\eta \psi \in X^{\theta}\right\} \stackrel{\text { onto }}{\rightarrow} Y^{0} .
\end{aligned}
$$

Since $\mathcal{A}_{(\theta)}^{*}: D\left(\mathcal{A}_{(\theta)}^{*}\right) \rightarrow Y^{0}$ and $\mathcal{J}_{(\theta)}: D\left(\mathcal{J}_{(\theta)}\right) \rightarrow Y^{0}$ are both closed operators in $Y^{0}$ and $X^{1} \times X^{\theta}$ is dense in $D\left(\mathcal{J}_{(\theta)}\right)$, we obtain from (12) that

$$
\mathcal{A}_{(\theta)}^{*}\left[\begin{array}{l}
\widetilde{u} \\
\tilde{v}
\end{array}\right]=\mathcal{J}_{(\theta)}\left[\begin{array}{l}
\tilde{u} \\
\tilde{v}
\end{array}\right],\left[\begin{array}{l}
\widetilde{u} \\
\tilde{v}
\end{array}\right] \in D\left(\mathcal{J}_{(\theta)}\right)
$$

By (i) and (iii) we next have $0 \in \rho\left(\mathcal{A}_{(\theta)}^{*}\right)$, which ensures in particular that the operator $\mathcal{A}_{(\theta)}^{*}$ is invertible. By this latter property and (13) it is clear that $D\left(\mathcal{A}_{(\theta)}^{*}\right)$ cannot have more elements than $D\left(\mathcal{J}_{(\theta)}\right)$. Part (v) is thus proved.

Part (vi) follows from the Lumer-Phillips theorem and from the observations concerning powers of accretive operators reported in [1, p. 164] (see also [8, p. 247]).

The sectoriality of $\mathcal{A}_{(\theta)}$ and its spectral properties mentioned in (vii) were reported in [5, Theorem 1.1]. Finally, compactness of $\left\{e^{-\mathcal{A}_{(\theta)} t}, t \geqslant 0\right\}$ for $\theta \neq 1$ is a consequence of (iv). Proposition 1 is thus proved.

REMARK 1. We draw attention to the fact that $\mathcal{A}_{(1)}$ does not have compact resolvent. This fact will assure that the semigroup $\left\{e^{\mathcal{A}_{(1)} t}, t \geqslant 0\right\}$ is not compact and makes this case specially interesting in the discussion of the asymptotics of (1). 
2.2. Partial Description of the fractional Power Scale for $\mathcal{A}_{(\theta)}, \theta$ $\in[1 / 2,1]$. Connecting the properties of $\mathcal{A}_{(\theta)}$ listed in Proposition 1 with the results of $[5,6]$ we obtain a partial description of the fractional power scale associated to $\mathcal{A}_{(\theta)}$. Before we can proceed we need the following general interpolation result:

Proposition 2. Let $\mathcal{X}_{i}, \mathcal{Y}_{i}, i=1,2$ be the Banach spaces such that

$$
\mathcal{X}_{1} \subset \mathcal{X}_{0}, \mathcal{Y}_{1} \subset \mathcal{Y}_{0}
$$

topologically and algebraically. Then,

$$
\left[\mathcal{X}_{0} \times \mathcal{Y}_{0}, \mathcal{X}_{1} \times \mathcal{Y}_{1}\right]_{\theta}=\left[\mathcal{X}_{0}, \mathcal{X}_{1}\right]_{\theta} \times\left[\mathcal{Y}_{0}, \mathcal{Y}_{1}\right]_{\theta}, \theta \in(0,1)
$$

Proof: The proof is an immediate consequence of the definition of complex interpolation spaces in [9, Section 1.9.2]).

Based on Proposition 2 it is now easy to get characterisations of the fractional power spaces $Y_{(\theta)}^{\alpha}, \alpha \in(0,1), \theta=1 / 2$ without referring to the general considerations of [6].

Proposition 3. For $\alpha \in[0,1]$ we have:

$$
Y_{(1 / 2)}^{\alpha}=D\left(\mathcal{A}_{(1 / 2)}^{\alpha}\right)=D\left(\left(\mathcal{A}_{(1 / 2)}^{*}\right)^{\alpha}\right)=X^{\alpha / 2+1 / 2} \times X^{\alpha / 2} .
$$

Proof: Recall that $Y_{(1 / 2)}^{1}=X^{1} \times X^{1 / 2}, Y^{0}=X^{1 / 2} \times X$ whereas, from Proposition 1 (vi),

$$
Y_{(1 / 2)}^{\alpha}=\left[Y^{0}, Y_{(1 / 2)}^{1}\right]_{\alpha}, \alpha \in(0,1) .
$$

Combining (17) and (15) we obtain

$$
Y_{(1 / 2)}^{\alpha}=\left[X^{1 / 2}, X^{1}\right]_{\alpha} \times\left[X, X^{1 / 2}\right]_{\alpha}, \alpha \in(0,1) .
$$

Next, by our assumptions on $A$, we have the equalities:

$$
\left[X^{1 / 2}, X^{1}\right]_{\alpha}=X^{1 / 2(1-\alpha)+\alpha},\left[X, X^{1 / 2}\right]_{\alpha}=X^{\alpha / 2},
$$

which justify the relations for (16) for $D\left(\mathcal{A}_{(1 / 2)}\right)$.

Since $\mathcal{A}_{(1 / 2)}$ is sectorial and has bounded imaginary powers, the same is true for $\mathcal{A}_{(1 / 2)}^{*}$ (see $\left[1\right.$, p. 13, p. 273]). Therefore, (17) holds for the powers of $\mathcal{A}^{*}$ as well. Recalling that $D\left(A_{(1 / 2)}\right)=D\left(A_{(1 / 2)}^{*}\right)=Y_{(1 / 2)}^{1}$ we get finally that

$$
Y_{(1 / 2)}^{\alpha}=D\left(\left(A_{(1 / 2)}^{*}\right)^{\alpha}\right), \alpha \in[0,1] .
$$

Proposition 3 is thus proved.

More generally we have: 
PROposition 4. For $\theta \in[1 / 2,1]$ the following equality holds:

(18)

$$
\begin{aligned}
Y_{(\theta)}^{\alpha} & =\left[Y^{0}, Y_{(\theta)}^{1}\right]_{\alpha} \\
& =\left\{\begin{array}{l}
X^{(1 / 2)+\alpha(1-\theta)} \times X^{\theta \alpha}, \alpha \in\left[0, \frac{1}{2}\right], \\
\left\{\left[\begin{array}{l}
\varphi \\
\psi
\end{array}\right] \in X^{(1 / 2)+\alpha(1-\theta)} \times X^{\theta-(1 / 2)+\alpha(1-\theta)}: A^{1-\theta} \varphi+\eta \psi \in X^{\theta \alpha}\right\}, \alpha \in\left[\frac{1}{2}, 1\right] .
\end{array}\right.
\end{aligned}
$$

Proof: The first equality in (18) follows from Proposition 1 (vi) and [9, Section 1.15.3]. The second equality in (18) results from [6, Theorem 1.1].

Recall that the extrapolation space $Y_{(\theta)_{-1}}$ of $Y^{0}$ generated by $\mathcal{A}_{(\theta)}$ is the completion of the normed space $\left(Y^{0},\left\|\mathcal{A}_{(\theta)}^{-1} \cdot\right\|_{Y^{0}}\right)$. We may infer that:

Proposition 5. Let $\theta \in[1 / 2,1]$ and $\mathcal{A}_{(\theta)_{-1}}$ denotes the closure of $\mathcal{A}_{(\theta)}$ in $Y_{(\theta)_{-1}}$. Then,

1. $\mathcal{A}_{(\theta)_{-1}}$ is sectorial and positive operator in the space $Y_{(\theta)_{-1}}$ with $D\left(\mathcal{A}_{(\theta)_{-1}}\right)$ $=Y_{(\theta)_{-1}}^{1}=Y^{0}$,

2. imaginary powers of $\mathcal{A}_{(\theta)-1}$ are bounded,

3. if $\theta \neq 1$, then $\mathcal{A}_{(\theta)_{-1}}$ has compact resolvent.

Proof: By $\left[1\right.$, p. 262] we know that $\rho\left(\mathcal{A}_{(\theta)_{-1}}\right)=\rho\left(\mathcal{A}_{(\theta)}\right)$ whereas the required estimate for the quantity $|\lambda|\left\|\left(\lambda-\mathcal{A}_{(\theta)_{-1}}\right)^{-1}\right\|_{\mathcal{L}\left(Y_{(\theta)_{-1}}, Y_{(\theta)-1}\right)}$ is a consequence of the estimate for the resolvent of $\mathcal{A}_{(\theta)}$ in $Y^{0}$. Indeed, since $Y_{(\theta)}^{1}$ is dense in $Y^{0}, \mathcal{A}_{(\theta)_{-1}}$ is a continuous extension of $\mathcal{A}_{(\theta)}$ onto $Y^{0}$ such that $\mathcal{A}_{(\theta)_{-1}}$ is an isometric isomorphism from $Y^{0}$ onto $Y_{(\theta)_{-1}}$. In particular, $\mathcal{A}_{(\theta)_{-1}}=\mathcal{A}_{(\theta)}$ on $D\left(\mathcal{A}_{(\theta)}\right)$ and $\left(\lambda-\mathcal{A}_{(\theta)_{-1}}\right)^{-1}=\left(\lambda-\mathcal{A}_{(\theta)}\right)^{-1}$ on $Y^{0}$. Therefore, by the sectoriality of $\mathcal{A}_{(\theta)}$ in $Y^{0}$ we obtain the estimate

$$
\begin{aligned}
\left\|\left(\lambda-\mathcal{A}_{(\theta)_{-1}}\right)^{-1}\left[\begin{array}{l}
u \\
v
\end{array}\right]\right\|_{Y_{(\theta)-1}} & =\left\|\mathcal{A}_{(\theta)}^{-1}\left(\lambda-\mathcal{A}_{(\theta)_{-1}}\right)^{-1}\left[\begin{array}{l}
u \\
v
\end{array}\right]\right\|_{Y^{0}} \\
& =\left\|\mathcal{A}_{(\theta)}^{-1}\left(\lambda-\mathcal{A}_{(\theta)}\right)^{-1}\left[\begin{array}{l}
u \\
v
\end{array}\right]\right\|_{Y^{0}} \\
& =\left\|\left(\lambda-\mathcal{A}_{(\theta)}\right)^{-1} \mathcal{A}_{(\theta)}^{-1}\left[\begin{array}{l}
u \\
v
\end{array}\right]\right\|_{Y^{0}} \\
& \leqslant \frac{C}{|\lambda|}\left\|\mathcal{A}_{(\theta)}^{-1}\left[\begin{array}{l}
u \\
v
\end{array}\right]\right\|_{Y^{0}} \\
& =\frac{C}{|\lambda|}\left\|\left[\begin{array}{l}
u \\
v
\end{array}\right]\right\|_{Y_{(\theta)-1}},\left[\begin{array}{l}
u \\
v
\end{array}\right] \in Y^{0} .
\end{aligned}
$$

The latter can be extended next to the elements of $Y_{(\theta)_{-1}}$ by the density of $Y^{0}$ in $Y_{(\theta)_{-1}}$. This justifies sectoriality of $\mathcal{A}_{(\theta)_{-1}}$ in $Y_{(\theta)_{-1}}$. 
The remaining assertions are the direct consequences of the results reported in $[\mathbf{1}$, p. 266, p. 264]. The proof is complete.

We shall next study (1) as a sectorial problem in $Y_{(\theta)_{-1}}, \theta \in[1 / 2,1]$; that is

$$
\frac{d}{d t}\left[\begin{array}{l}
u \\
v
\end{array}\right]+\mathcal{A}_{(\theta)_{-1}}\left[\begin{array}{l}
u \\
v
\end{array}\right]=\mathcal{F}\left(\left[\begin{array}{l}
u \\
v
\end{array}\right]\right), t>0,\left[\begin{array}{l}
u \\
v
\end{array}\right]_{t=0}=\left[\begin{array}{l}
u_{0} \\
v_{0}
\end{array}\right]
$$

Our concern will be the solutions to (19) originating at the elements of $Y^{0}=D\left(\mathcal{A}_{(\theta)_{-1}}\right)$ $=Y_{(\theta)_{-1}}^{1}$ and therefore we shall be working in the critical case. To that end we shall need to know sharp embeddings of the fractional power spaces into known spaces.

The embeddings below relate the spaces in the interpolated-extrapolated fractional power scale and known spaces. They are crucial in obtaining the $\varepsilon$-regular properties for the nonlinearities in (19).

Lemma 1. Let $\left[\left(X^{\alpha}, A_{\alpha}\right), \alpha \in \mathbb{R}\right]\left(A_{\alpha}\right.$ being the realisation of $A$ in $\left.X^{\alpha}\right)$ be generated by $\left(L^{2}(\Omega),\left(-\Delta_{D}\right)\right)$. Then the following embeddings hold:

$$
\begin{aligned}
& \left\{\begin{array}{l}
Y_{(\theta)_{-1}}^{1+\alpha} \subset H^{1+2 \alpha(1-\theta)}(\Omega) \times H^{2 \theta \alpha}(\Omega) \subset L^{q_{1}}(\Omega) \times L^{q_{2}}(\Omega), \\
\text { for } 1 \leqslant q_{1} \leqslant \frac{2 n}{n-2-4 \alpha(1-\theta)}, 1 \leqslant q_{2} \leqslant \frac{2 n}{n-4 \alpha \theta} \\
\alpha \in[0,1 / 2], \theta \in[1 / 2,1], n \geqslant 3, \text { and } \alpha(1-\theta)<\frac{1}{4} \text { if } n=3,
\end{array}\right. \\
& \left\{\begin{array}{l}
Y_{(\theta)-1}^{\alpha} \supset X^{(1 / 2)-(1-\alpha)(1-\theta)} \times X^{-(1 / 2)+\alpha(1-\theta)} \supset X^{(1 / 2)-(1-\alpha)(1-\theta)} \times L^{q}(\Omega), \\
q \geqslant \frac{2 n}{n+2-4 \alpha(1-\theta)}, \alpha \in\left[0, \frac{1}{2}\right], \theta \in\left[\frac{1}{2}, 1\right], n \geqslant 3 .
\end{array}\right.
\end{aligned}
$$

Proof: To obtain (20) we use interpolation and the results from (18)

$Y_{(\theta)_{-1}}^{1+\alpha}=\left[Y_{(\theta)_{-1}}^{1}, Y_{(\theta)_{-1}}^{2}\right]_{\alpha}=\left[Y_{(\theta)}^{0}, Y_{(\theta)}^{1}\right]_{\alpha}=Y_{(\theta)}^{\alpha}=X^{(1 / 2)+\alpha(1-\theta)} \times X^{\theta \alpha}, \alpha \in\left[0, \frac{1}{2}\right], \theta \in\left[\frac{1}{2}, 1\right]$.

Therefore,

$$
Y_{(\theta)_{-1}}^{1+\alpha} \subset H^{1+2 \alpha(1-\theta)}(\Omega) \times H^{2 \theta \alpha}(\Omega) \subset L^{q_{1}}(\Omega) \times L^{q_{2}}(\Omega)
$$

whenever

$$
1 \leqslant q_{1} \leqslant \frac{2 n}{n-2-4 \alpha(1-\theta)}, \quad 1 \leqslant q_{2} \leqslant \frac{2 n}{n-4 \theta \alpha},
$$

where $\alpha \in[0,1 / 2], \theta \in[1 / 2,1], n \geqslant 3$.

To prove (21) we first note that, based on [1, p. 267, Corollary 1.3.9],

$$
\left(\mathcal{A}_{(\theta)_{-1}}\right)^{-1+|\beta|}: Y_{(\theta)_{-1}} \stackrel{\substack{\text { isometric } \\ \text { isomorphism }}}{\longrightarrow} Y_{(\theta)_{-|\beta|}},-1 \leqslant \beta \leqslant 0
$$

so that in particular

$$
Y_{(\theta)_{-(1-\alpha)}}=Y_{(\theta)_{-1}}^{\alpha}, 0 \leqslant \alpha \leqslant 1
$$


We now focus on the case $\alpha \in[0,1 / 2]$.

From (22) and [1, p. 266, Theorem 1.3.8] we obtain

$$
\begin{aligned}
& \left\|\left[\begin{array}{l}
\varphi \\
\psi
\end{array}\right]\right\|_{Y_{(\theta)-1}^{\alpha}}=\left\|\left[\begin{array}{l}
\varphi \\
\psi
\end{array}\right]\right\|_{Y_{(\theta)_{-(1-\alpha)}}}=\left\|\mathcal{A}_{(\theta)}^{-(1-\alpha)}\left[\begin{array}{l}
\varphi \\
\psi
\end{array}\right]\right\|_{Y^{0}}=\left\|\mathcal{A}_{(\theta)}^{\alpha} \mathcal{A}_{(\theta)}^{-1}\left[\begin{array}{l}
\varphi \\
\psi
\end{array}\right]\right\|_{Y^{0}}=\left\|\mathcal{A}_{(\theta)}^{-1}\left[\begin{array}{l}
\varphi \\
\psi
\end{array}\right]\right\|_{Y_{(\theta)}^{\alpha}} \\
& =\left\|\mathcal{A}_{(\theta)}^{-1}\left[\begin{array}{l}
\varphi \\
\psi
\end{array}\right]\right\|_{X^{(1 / 2)+\alpha(1-\theta)} \times X^{\theta \alpha}}=\left\|\left[\begin{array}{c}
\eta A^{-(1-\theta)} \varphi+A^{-1} \psi \\
-\varphi
\end{array}\right]\right\|_{X^{(1 / 2)+\alpha(1-\theta) \times X^{\theta \alpha}}} \\
& =\left\|A^{(1 / 2)+\alpha(1-\theta)}\left(\eta A^{-(1-\theta)} \varphi+A^{-1} \psi\right)\right\|_{X^{0}}+\left\|A^{\theta \alpha} \varphi\right\|_{X^{0}} \\
& =\left\|\eta A^{(1 / 2)-(1-\alpha)(1-\theta)} \varphi+A^{-(1 / 2)+\alpha(1-\theta)} \psi\right\|_{X^{0}}+\left\|A^{\theta \alpha} \varphi\right\|_{X^{0}},\left[\begin{array}{l}
\varphi \\
\psi
\end{array}\right] \in Y^{0},
\end{aligned}
$$

$\alpha \in[0,1 / 2], \theta \in[1 / 2,1]$.

Consequently, since

$$
\frac{1}{2}-(1-\alpha)(1-\theta) \geqslant \theta \alpha \text { for } \alpha \in\left[0, \frac{1}{2}\right], \theta \in\left[\frac{1}{2}, 1\right]
$$

we obtain

$$
\begin{aligned}
\left\|\left[\begin{array}{l}
\varphi \\
\psi
\end{array}\right]\right\|_{Y_{(\theta)-1}^{\alpha}} & \leqslant c\left(\left\|A^{(1 / 2)-(1-\alpha)(1-\theta)} \varphi\right\|_{X^{0}}+\left\|A^{-(1 / 2)+\alpha(1-\theta)} \psi\right\|_{X^{0}}\right) \\
& =c\left\|\left[\begin{array}{l}
\varphi \\
\psi
\end{array}\right]\right\|_{X^{(1 / 2)-(1-\alpha)(1-\theta)} \times X^{-(1 / 2)+\alpha(1-\theta)}},\left[\begin{array}{c}
\varphi \\
\psi
\end{array}\right] \in Y^{0}, \alpha \in\left[0, \frac{1}{2}\right], \theta \in\left[\frac{1}{2}, 1\right] .
\end{aligned}
$$

By the density of $Y^{0}$ in $X^{(1 / 2)-(1-\alpha)(1-\theta)} \times X^{-(1 / 2)+\alpha(1-\theta)}$, this proves the first inclusion in (21).

The second inclusion in (21) is a consequence of a duality argument and the inclusions

$$
\left(X^{-(1 / 2)+\alpha(1-\theta)}\right)^{*}=X^{(1 / 2)-\alpha(1-\theta)} \subset H^{1-2 \alpha(1-\theta)}(\Omega) \subset L^{p}(\Omega)
$$

for $1 \leqslant p \leqslant((2 n) / n-2+4 \alpha(1-\theta))$. The proof is now completed.

In the case $\theta=1 / 2$ we can describe more completely the fractional power scale.

\subsection{Complete Description of the Fractional Power Scale for $\mathcal{A}_{(1 / 2)}$.} Based on $[1$, Chapter V] we shall describe here the extrapolated fractional power scale of order 1 corresponding to a pair $\left(Y^{0}, \mathcal{A}_{(1 / 2)}\right)$.

LEMMA 2. Let $Y_{(1 / 2)_{-1}}$ denote the extrapolation space of $Y^{0}$ generated by $\mathcal{A}_{(1 / 2)}$. The following equality holds:

$$
Y_{(1 / 2)_{-1}}=X \times X^{-1 / 2}
$$


PROOF: Recall first that $Y_{(1 / 2)_{-1}}$ is the completion of the normed space $\left(Y^{0}, \| \mathcal{A}_{(1 / 2)}^{-1}\right.$. $\left.\|_{Y^{0}}\right)$. Since

$$
\left\|\mathcal{A}_{(1 / 2)}^{-1}\left[\begin{array}{l}
u \\
v
\end{array}\right]\right\|_{Y^{0}} \leqslant c\left\|\left[\begin{array}{l}
u \\
v
\end{array}\right]\right\|_{X \times X^{-1 / 2}} \leqslant c^{\prime}\left\|\mathcal{A}_{(1 / 2)}^{-1}\left[\begin{array}{l}
u \\
v
\end{array}\right]\right\|_{Y^{0}},\left[\begin{array}{l}
u \\
v
\end{array}\right] \in Y^{0},
$$

the completions of $\left(Y^{0},\left\|\mathcal{A}_{(1 / 2)}^{-1} \cdot\right\|_{Y^{0}}\right)$ and $\left(Y^{0},\|\cdot\|_{X \times X^{-1 / 2}}\right)$ coincide. Recalling that $Y^{0}=X^{1 / 2} \times X$, we obtain (23). The proof is complete.

THEOREM 2. The following characterisation holds:

$$
Y_{(1 / 2)-1}^{k+\alpha}=X^{(k+\alpha) / 2} \times X^{(k-1+\alpha) / 2}, \alpha \in[0,1], k \in \mathbb{N},
$$

Proof: By Proposition 5 the usual interpolation arguments may be used so that for $k=1,2, \ldots$, we get

$$
\begin{aligned}
Y_{(1 / 2)_{-1}}^{k+\alpha} & =\left[Y_{(1 / 2)_{-1}}^{k}, Y_{(1 / 2)_{-1}}^{k+1}\right]_{\alpha}=\left[Y_{(1 / 2)}^{k-1}, Y_{(1 / 2)}^{k}\right]_{\alpha} \\
& =\left[X^{k / 2} \times X^{(k-1) / 2}, X^{(k+1) / 2} \times X^{k / 2}\right]_{\alpha} \\
& =\left[X^{k / 2}, X^{(k+1) / 2}\right]_{\alpha} \times\left[X^{(k-1) / 2}, X^{k / 2}\right]_{\alpha}=X^{(k+\alpha) / 2} \times X^{(k-1+\alpha) / 2} .
\end{aligned}
$$

For $k=0$ instead of the standard arguments above we also need the properties of the Hilbert scale $\left[X^{\alpha}, \alpha \in \mathbb{R}\right]$ (see $[1$, p. 274]) as well as the duality theory for the complex interpolation method (see [9, Theorem 1.11.3]). Identifying $X$ with its dual $X^{*}$ we then have:

$$
\begin{aligned}
Y_{(1 / 2)_{-1}}^{\alpha} & =\left[Y_{(1 / 2)_{-1}}, Y_{(1 / 2)_{-1}}^{1}\right]_{\alpha}=\left[Y_{(1 / 2)_{-1}}, Y^{0}\right]_{\alpha} \\
& =\left[X \times X^{-1 / 2}, X^{1 / 2} \times X\right]_{\alpha}=\left[X, X^{1 / 2}\right]_{\alpha} \times\left[X^{-1 / 2}, X\right]_{\alpha} \\
& =X^{\alpha / 2} \times\left[\left(\left(X^{*}\right)^{1 / 2}\right)^{*}, X^{*}\right]_{\alpha}=X^{\alpha / 2} \times\left[X^{1 / 2}, X\right]_{\alpha}^{*}=X^{\alpha / 2} \times\left[X, X^{1 / 2}\right]_{1-\alpha}^{*} \\
& =X^{\alpha / 2} \times\left(X^{(1-\alpha) / 2}\right)^{*}=X^{\alpha / 2} \times X^{(\alpha-1) / 2}
\end{aligned}
$$

The proof is complete.

Using Theorem 2 it is easy to enlarge the admissible range of $\alpha$ in the inclusions (20), (21). Also, in the case $\alpha=1 / 2$ the proof of the embeddings is much simpler.

Corollary 1. (Embedding of spaces $\left.Y_{(1 / 2)_{-1}}^{1+\alpha}\right)$. Let $\left[\left(X^{\alpha}, A_{\alpha}\right), \alpha \in \mathbb{R}\right]$ be the scale generated by $\left(L^{2}(\Omega),\left(-\Delta_{D}\right)\right)$. Then the following embeddings hold:

$$
\begin{gathered}
\left\{\begin{array}{l}
Y_{(1 / 2)-1}^{1+\alpha} \subset H^{1+\alpha}(\Omega) \times H^{\alpha}(\Omega) \subset L^{q_{1}}(\Omega) \times L^{q_{2}}(\Omega) \\
\text { for } 1 \leqslant q_{1} \leqslant
\end{array}\right. \\
\qquad \begin{array}{l}
X^{\alpha / 2} \times L^{q}(\Omega) \subset Y_{(1 / 2)-1}^{\alpha} \\
\text { for } \frac{2 n}{n+2-2 \alpha} \leqslant q, \alpha \in[0,1] .
\end{array}
\end{gathered}
$$


PROOF: The proof of (25) follows from the interpolation result for the spaces of Bessel potentials (see [9, Theorem 2, Section 4.3.1]) and Sobolev Embedding Theorem (see [9, Theorem 4.6.1]). Condition (26) is a consequence of the duality argument and the inclusions

$$
\left(X^{(\alpha-1) / 2}\right)^{*}=X^{(1-\alpha) / 2} \subset H^{1-\alpha}(\Omega) \subset L^{p}(\Omega) \text { for } 1 \leqslant p \leqslant(2 n / n+2 \alpha-2), \alpha \in[0,1],
$$

where $X$ was identified with its dual $X^{*}$. The proof is complete.

REMARK 2. Following the terminology of $[1$, p. 258, p. 266],

$$
\left[\left(X^{\sigma / 2} \times X^{(\sigma-1) / 2},\left(\mathcal{A}_{(1 / 2)_{-1}}\right)_{\sigma}\right), \sigma \in[0,+\infty)\right]
$$

is a compactly injected one-sided fractional power scale generated by $\left(Y_{(1 / 2)_{-1}}, \mathcal{A}_{(1 / 2)_{-1}}\right)$. Simultaneously

$$
\left[\left(X^{(\sigma+1) / 2} \times X^{\sigma / 2},\left(\mathcal{A}_{(1 / 2)_{-1}}\right)_{\sigma+1}\right), \sigma \in[-1,+\infty)\right]
$$

is the extrapolated fractional power scale of first order generated by $\left(Y^{0}, \mathcal{A}_{(1 / 2)}\right)$.

\section{Solvability of (1) in $Y^{0}$}

Here we employ the results of [2] to obtain local well posedness and regularity results for (2) with initial conditions in $Y^{0}$ and the nonlinearities $f$ growing critically. We begin with the concept of $\varepsilon$-regular maps and prove that under certain growth conditions the map $\mathcal{F}$ can be decomposed as a sum of $\varepsilon$-regular maps. Next we use this fact to obtain a local existence result for $\varepsilon$-regular solutions to (1).

Following the results of Section 2 we are going to study (1) as the sectorial problem (19) in a Hilbert space $Y_{(\theta)_{-1}}$, and look for the solutions to (19) originating at the elements of $Y^{0}$.

For this we need first recall the notions of the $\varepsilon$-regular maps and $\varepsilon$-regular solutions to (19) (see [2], [3]).

3.1. $\varepsilon$-REgULAR MAPS AND $\varepsilon$-REgULAR SOLUTIONS. Let $P$ be a sectorial, positive operator acting in a Banach space $Z=Z^{0}$ and $\varepsilon$ be a nonnegative number.

DEFinition 1: $\quad G: D(G) \rightarrow Z$ is said to be an $\varepsilon$-regular map relatively to $\left(Z^{1}, Z^{0}\right)$ (or, equivalently, $G$ is said to belong to a subclass $\mathcal{F}(\varepsilon, \rho, \gamma(\varepsilon), C)$ of nonlinearities acting in $Z^{0}$ ) if and only if there are constants $\rho>1, \gamma(\varepsilon) \geqslant 0, C>0$ such that

1. $\rho \varepsilon \leqslant \gamma(\varepsilon)<1$,

2. $G$ takes $Z^{1+\varepsilon}$ into $Z^{\gamma(\varepsilon)}$,

3. $G$ satisfies the estimate 


$$
\left\|G\left(z_{1}\right)-G\left(z_{2}\right)\right\|_{Z^{\gamma+\varepsilon}} \leqslant C\left\|z_{1}-z_{2}\right\|_{Z^{1+\varepsilon}}\left(\left\|z_{1}\right\|_{Z^{1+\varepsilon}}^{\rho-1}+\left\|z_{2}\right\|_{Z^{1+\varepsilon}}^{\rho-1}+1\right), z_{1}, z_{2} \in Z^{1+\varepsilon} .
$$

Consider next the abstract Cauchy problem

$$
\dot{z}+P z=G(z), t>0, z(0)=z_{0} .
$$

Definition 2: Let $\varepsilon \geqslant 0, \tau>0, z_{0} \in Z^{1}$, and $z=z\left(\cdot, z_{0}\right):[0, \tau] \rightarrow Z^{1}$. We say that $z$ is an $\varepsilon$-regular solution to (28) if and only if

1. $z \in C\left([0, \tau], Z^{1}\right) \cap C\left((0, \tau], Z^{1+\varepsilon}\right)$,

2. $z$ satisfies the Cauchy integral formula:

$$
z(t)=e^{-P t} z_{0}+\int_{0}^{t} e^{-P(t-s)} G(z(s)) d s, z \in[0, \tau] .
$$

The existence of an $\varepsilon$-regular solutions was proved in [2]. The result below comes from [3] and is an extension of the original theorem reported in [2].

Proposition 6. Let $\widetilde{z}_{0} \in Z^{1}$ and $B_{Z^{1}}\left(\widetilde{z}_{0}, r\right)$ denote a ball in $Z^{1}$ with radius $r>0$ centred at $\tilde{z}_{0}$.

Assume that $G=\sum_{i=1}^{k} G_{i}$ and, for $1 \leqslant i \leqslant k, G_{i}$ belongs to the class $\mathcal{F}\left(\varepsilon_{i}, \rho_{i}, \gamma_{i}\left(\varepsilon_{i}\right), C_{i}\right)$ with certain $\varepsilon_{i}>0$. Suppose also that

$$
\min \left\{\gamma_{i}\left(\varepsilon_{i}\right) ; 1 \leqslant i \leqslant n\right\}=: \widetilde{\gamma}>\widetilde{\varepsilon}:=\max \left\{\varepsilon_{i} ; 1 \leqslant i \leqslant n\right\} .
$$

Then, there are $r>0$ and $\tau_{0}>0$ such that for each $z_{0} \in B_{Z^{1}}\left(\widetilde{z}_{0}, r\right)$ there exists a unique $\widetilde{\varepsilon}$-regular solution $z=z\left(\cdot, z_{0}\right)$ to (28). In addition,

(i) $t^{\varsigma}\left\|z\left(t, z_{0}\right)\right\|_{Z^{1+\zeta}} \rightarrow 0$ as $t \rightarrow 0^{+}, 0<\zeta<\tilde{\gamma}$,

(ii) $t^{\zeta}\left\|z\left(t, z_{1}\right)-z\left(t, z_{2}\right)\right\|_{2^{1+\zeta}} \leqslant C^{\prime}\left\|z_{1}-z_{2}\right\|_{Z^{1}}, t \in\left[0, \tau_{0}\right], \quad 0 \leqslant \zeta \leqslant \zeta_{0}<\tilde{\gamma}$, $z_{1}, z_{2} \in B_{Z^{1}}\left(\widetilde{z}_{0}, r\right)$

(iii) $z \in C\left(\left(0, \tau_{0}\right], Z^{1+\tilde{\gamma}}\right) \cap C^{1}\left(\left(0, \tau_{0}\right], Z^{1+\zeta}\right), 0 \leqslant \zeta<\tilde{\gamma}$, and, in particular, $z$ satisfies both relations in (28).

We are now fully prepared to prove the solvability of (19) in $Y^{0}$.

3.2. LOCAL WELL POSEDNESS FOR (19). To make the presentation easier let us assume that $f$ in (1) is such that

$$
f(u, v)=f_{1}(u)+f_{2}(v), f_{i}: \mathbb{R} \rightarrow \mathbb{R}, i=1,2 .
$$

We shall require $f_{i}, \mathrm{i}=1,2$, fulfill the estimate:

$$
\left|f_{i}\left(s_{1}\right)-f_{i}\left(s_{2}\right)\right| \leqslant c\left|s_{1}-s_{2}\right|\left(\left|s_{1}\right|^{\rho_{i}-1}+\left|s_{2}\right|^{\rho_{i}-1}+1\right), s_{1}, s_{2} \in \mathbb{R},
$$


with certain $\rho_{i} \in(1,+\infty), i=1,2$. Of course, condition (31) is much stronger than the assumption of local Lipschitz continuity of $f$. As we shall see that once we have proved local well posedness results for (1) with $f$ satisfying (30) and (31), we can easily extend them to functions $f$ satisfying (7).

REMARK 3. Of course the assumption $\rho_{i} \neq 1$ is merely technical. If (31) is satisfied with $\rho_{i}=1$, it is obvious that (31) holds also with arbitrary $\rho_{i}>1$.

The next two results, Lemmas 3 and 4 , are fundamental to proving the $\varepsilon$-regular properties of the nonlinearities in (19).

Lemma 3. Let $\theta \in[1 / 2,1]$ and

$$
\left|f_{1}\left(s_{1}\right)-f_{1}\left(s_{2}\right)\right| \leqslant c\left|s_{1}-s_{2}\right|\left(1+\left|s_{1}\right|^{\rho_{1}-1}+\left|s_{2}\right|^{\rho_{1}-1}\right), s_{1}, s_{2} \in \mathbb{R},
$$

where

$$
1<\rho_{1} \leqslant \frac{n+2}{n-2}=\rho_{1}(n)
$$

Then, for each $\varepsilon \in\left[0,\left(1 /\left(2 \rho_{1}\right)\right)\right]$ there is a certain $\gamma(\varepsilon) \in\left[\rho_{1} \varepsilon,(1 / 2)\right]$ such that

$$
\begin{aligned}
& \left\|f_{1}\left(w_{1}\right)-f_{1}\left(w_{2}\right)\right\|_{L^{(2 n) /(n+2-4 \gamma(\epsilon)(1-\theta))}} \\
& \leqslant c\left\|w_{1}-w_{2}\right\|_{H^{1+2 \epsilon(1-\theta)}}\left(1+\left\|w_{1}\right\|_{H^{1+2 \varepsilon(1-\theta)}}^{\rho_{1}-1}+\left\|w_{2}\right\|_{H^{1+2 e(1-\theta)}}^{\rho_{1}-1}\right) \\
& w_{1}, w_{2} \in H^{1+2 \epsilon(1-\theta)}(\Omega) .
\end{aligned}
$$

Furthermore;

1. if $\theta \in[1 / 2,1)$ and $\rho_{1}=\rho_{1}(n)$ we necessarily have $\gamma(\varepsilon)=\rho_{1} \varepsilon$ and

2. if $\theta=1$ we can take $\varepsilon=0$ and $\gamma(0)=1 / 2$.

Proof: From (32), the Hölder inequality and the Sobolev embedding we obtain:

$$
\begin{aligned}
& \left\|f_{1}\left(w_{1}\right)-f_{1}\left(w_{2}\right)\right\|_{L^{(2 n) /(n+2-4 \gamma(e)(1-\theta))(\Omega)}} \\
& \leqslant c\left[\int_{\Omega}\left[\left|w_{1}-w_{2}\right|\left(1+\left|w_{1}\right|^{\rho_{1}-1}+\left|w_{2}\right|^{\rho_{1}-1}\right)\right]^{(2 n) /(n+2-4 \gamma(\varepsilon)(1-\theta))}\right]^{(n+2-4 \gamma(\varepsilon)(1-\theta)) /(2 n)} \\
& \leqslant c\left[\int_{\Omega}\left|w_{1}-w_{2}\right|^{(2 n) /(n-2-4 \varepsilon(1-\theta))}\right]^{(n-2-4 \varepsilon(1-\theta)) /(2 n)} \\
& \times\left[\int_{\Omega}\left(1+\left|w_{1}\right|^{\rho_{1}-1}+\left|w_{2}\right|^{\rho_{1}-1}\right)^{(2 n) /(4+4(\varepsilon-\gamma(\varepsilon))(1-\theta))}\right]^{(4+4(\varepsilon-\gamma(\varepsilon))(1-\theta)) /(2 n)} \\
& \leqslant c\left\|w_{1}-w_{2}\right\|_{L^{(2 n) /(n-2-4 \varepsilon(1-\theta))(\Omega)}}\left\|1+\left|w_{1}\right|^{\rho_{1}-1}+\left|w_{2}\right|^{\rho_{1}-1}\right\|_{L^{(2 n) /(4+4(\varepsilon-\gamma(\varepsilon))(1-\theta))(\Omega)}} \\
& \leqslant \widetilde{c}\left\|w_{1}-w_{2}\right\|_{L^{(2 n) /(n-2-4 \varepsilon(1-\theta))(\Omega)}}\left(1+\left\|w_{1}\right\|_{L^{\left(2 n\left(\rho_{1}-1\right)\right) /(4+4(\varepsilon-\gamma(\varepsilon))(1-\theta))}(\Omega)}^{\rho_{1}-1}\right. \\
& \left.+\left\|w_{2}\right\|_{L^{\left(2 n\left(\rho_{1}-1\right)\right) /(4+4(e-\gamma(e))(1-\theta))}(\Omega)}^{\rho_{1}-1}\right) .
\end{aligned}
$$


Since

$$
L^{(2 n) /(n-2-4 \varepsilon(1-\theta))}(\Omega) \supset H^{1+2 \varepsilon(1-\theta)}(\Omega)
$$

we only need to guarantee that

$$
L^{\left(2 n\left(\rho_{1}-1\right)\right) /(4+4(\varepsilon-\gamma(\varepsilon))(1-\theta))}(\Omega) \supset H^{1+2 \varepsilon(1-\theta)}(\Omega) .
$$

This will be true provided that

$$
\frac{2 n\left(\rho_{\mathrm{l}}-1\right)}{4+4(\varepsilon-\gamma(\varepsilon))(1-\theta)} \leqslant \frac{2 n}{n-2-4 \varepsilon(1-\theta)}
$$

which implies the inequality

$$
\rho_{1} \leqslant \frac{n+2-4 \gamma(\varepsilon)(1-\theta)}{n-2-4 \varepsilon(1-\theta)}
$$

Because $\gamma(\varepsilon) \geqslant \rho_{1} \varepsilon$, we have

$$
\rho_{1} \leqslant \frac{n+2-4 \rho_{1} \varepsilon(1-\theta)}{n-2-4 \varepsilon(1-\theta)} .
$$

The above condition ensures that $\rho_{1} \leqslant(n+2) /(n-2)=\rho_{1}(n)$.

It also shows that, if $\theta \neq 1$, then $\rho_{1}(n)$ cannot be attained for $\gamma(\varepsilon)>\rho_{1} \varepsilon$. The proof is now completed.

Corollary 2. Assume that $f_{1}$ satisfies (32) with $1<\rho_{1} \leqslant(n+2) /(n-2)$ and $\theta \in[1 / 2,1]$. Let $\mathcal{F}_{1}$ be the map defined by

$$
\mathcal{F}_{1}\left(\left[\begin{array}{l}
u \\
v
\end{array}\right]\right)=\left[\begin{array}{c}
0 \\
F_{1}(u)
\end{array}\right]
$$

where $F_{1}(u)$ is the Nemitskin map associated with $f_{1}$. Then, $\mathcal{F}_{1}$ is an $\varepsilon$-regular map relative to $\left(Y_{(\theta)_{-1}}^{1}, Y_{(\theta)_{-1}}\right)$ for each $\varepsilon \in\left[0,\left(1 / 2 \rho_{1}\right)\right]$ and $\gamma(\varepsilon)=\rho_{1} \varepsilon$. That is,

$$
\begin{aligned}
& \left\|\mathcal{F}_{1}\left(\left[\begin{array}{l}
u \\
v
\end{array}\right]\right)-\mathcal{F}_{1}\left(\left[\begin{array}{l}
u^{\prime} \\
v^{\prime}
\end{array}\right]\right)\right\|_{Y_{(\theta)_{-1}}^{\gamma(e)}} \\
& \leqslant c\left\|\left[\begin{array}{l}
u \\
v
\end{array}\right]-\left[\begin{array}{l}
u^{\prime} \\
v^{\prime}
\end{array}\right]\right\|_{Y_{(\theta)_{-1}^{1}}^{1+\varepsilon}}\left(1+\left\|\left[\begin{array}{l}
u \\
v
\end{array}\right]\right\|_{Y_{(\theta)_{-1}^{1}}^{1+\varepsilon}}^{\rho_{1}-1}+\left\|\left[\begin{array}{l}
u^{\prime} \\
v^{\prime}
\end{array}\right]\right\|_{Y_{(\theta)_{-1}}^{1+\varepsilon}}^{\rho_{1}-1}\right),\left[\begin{array}{l}
u \\
v
\end{array}\right],\left[\begin{array}{c}
u^{\prime} \\
v^{\prime}
\end{array}\right] \in Y_{(\theta)_{-1}}^{1+\varepsilon},
\end{aligned}
$$

whenever $\varepsilon \in\left[0,\left(1 /\left(2 \rho_{1}\right)\right)\right]$.

The intervals of $\varepsilon$-regularity of $\mathcal{F}_{1}$ are a function of the growth exponent $\rho_{1}$. In Figure 1 the reader can see how the intervals of $\varepsilon$-regularity change as a function of $\rho_{1}$.

The $u$-coordinate of $\mathcal{F}\left(\mathcal{F}_{1}\right.$ above) behaves very much the same in the case $\theta=1 / 2$ and in the case $\theta \in(1 / 2,1]$. As for the $v$-coordinate of $\mathcal{F}\left(\mathcal{F}_{2}\right.$ below), in the case $\theta \in(1 / 2,1]$ it has completely different behaviour from that observed in the case $\theta=1 / 2$. For the case $\theta \in(1 / 2,1]$ the behaviour of the $v$-coordinate of $\mathcal{F}$ resembles the case identified as an ultra-critical map in [2]. That can be seen from the following lemma. 


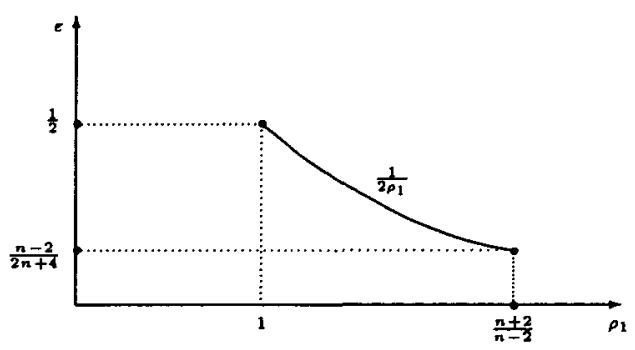

Figure 1: Intervals of $\varepsilon$-regularity for $\mathcal{F}_{1}$

LEMMA 4. Let $\theta \in[1 / 2,1]$ and

$$
\left|f_{2}\left(s_{1}\right)-f_{2}\left(s_{2}\right)\right| \leqslant c\left|s_{1}-s_{2}\right|\left(1+\left|s_{1}\right|^{\rho_{2}-1}+\left|s_{2}\right|^{\rho_{2}-1}\right), s_{1}, s_{2} \in \mathbb{R}
$$

where

$$
1<\rho_{2} \leqslant \frac{n+4 \theta}{n}=\rho_{2}(n, \theta) .
$$

Define $\varepsilon_{0}=(n) /(2 n+8 \theta)$. Then, there exist $\varepsilon \in\left[0, \varepsilon_{0}\right]$ and $\gamma(\varepsilon) \in\left[\rho_{2} \varepsilon, 1 / 2\right]$ such that

$$
\begin{aligned}
& \left\|f_{2}\left(w_{1}\right)-f_{2}\left(w_{2}\right)\right\|_{L^{(2 n) /(n+2-4 \gamma(\varepsilon)(1-\theta))}(\Omega)} \\
& \quad \leqslant c\left\|w_{1}-w_{2}\right\|_{H^{2 \varepsilon \theta}(\Omega)}\left(1+\left\|w_{1}\right\|_{H^{2 \varepsilon \theta}(\Omega)}^{\rho_{2}-1}+\left\|w_{2}\right\|_{H^{2 \varepsilon \theta}(\Omega)}^{\rho_{2}-1}\right), w_{1}, w_{2} \in H^{2 \varepsilon \theta}(\Omega) .
\end{aligned}
$$

Proof: From (37), the Hölder inequality and the Sobolev embedding we obtain:

$$
\begin{aligned}
& \left\|f_{2}\left(w_{1}\right)-f_{2}\left(w_{2}\right)\right\|_{L^{(2 n) /(n+2-4 \gamma(\varepsilon)(1-\theta))(\Omega)}} \\
& \leqslant c\left[\int_{\Omega}\left[\left|w_{1}-w_{2}\right|\left(1+\left|w_{1}\right|^{\rho_{2}-1}+\left|w_{2}\right|^{\rho_{2}-1}\right)\right]^{(2 n) /(n+2-4 \gamma(\varepsilon)(1-\theta))}\right]^{(n+2-4 \gamma(\varepsilon)(1-\theta)) /(2 n)} \\
& \leqslant c\left[\int_{\Omega}\left|w_{1}-w_{2}\right|^{(2 n) /(n-4 \varepsilon \theta)}\right]^{(n-4 \varepsilon \theta) /(2 n)} \\
& \times\left[\int_{\Omega}\left(1+\left|w_{1}\right|^{\rho_{2}-1}+\left|w_{2}\right|^{\rho_{2}-1}\right)^{(2 n) /(2-4 \gamma(\varepsilon)(1-\theta)+4 \varepsilon \theta)}\right]^{(2-4 \gamma(\varepsilon)(1-\theta)+4 \varepsilon \theta) /(2 n)} \\
& \leqslant c\left\|w_{1}-w_{2}\right\|_{L^{(2 n) /(n-4 \varepsilon \theta)}(\Omega)}\left\|1+\left|w_{1}\right|^{\rho_{2}-1}+\left|w_{2}\right|^{\rho_{2}-1}\right\|_{L^{(2 n) /(2-4 \gamma(\varepsilon)(1-\theta)+4 \varepsilon \theta)(\Omega)}} \\
& \leqslant \widetilde{c}\left\|w_{1}-w_{2}\right\|_{L^{(2 n) /(n-4 \epsilon \theta)}(\Omega)}\left(1+\left\|w_{1}\right\|_{L^{\left(2 n\left(\rho_{2}-1\right)\right) /(2-4 \gamma(\varepsilon)(1-\theta)+4 \varepsilon \theta)(\Omega)}}^{\rho_{2}-1}\right. \\
& \left.+\left\|w_{2}\right\|_{L^{\left(2 n\left(\rho_{2}-1\right)\right) /(2-4 \gamma(\varepsilon)(1-\theta)+4 \varepsilon \theta)}(\Omega)}^{\rho_{2}-1}\right) \text {. }
\end{aligned}
$$

Therefore, (39) will be true provided that

$$
\frac{2 n\left(\rho_{2}-1\right)}{2-4 \gamma(\varepsilon)(1-\theta)+4 \varepsilon \theta} \leqslant \frac{2 n}{n-4 \varepsilon \theta}
$$


which implies both the condition

$$
\rho_{2} \leqslant \frac{n+2-4 \gamma(\varepsilon)(1-\theta)}{n-4 \varepsilon \theta}
$$

and, if we make use of the condition $\gamma(\varepsilon) \geqslant \rho_{2} \varepsilon$, also the inequality

$$
\rho_{2} \leqslant \frac{n+2}{n-8 \varepsilon(\theta-(1 / 2))} .
$$

Since the right hand side of (41) attains the value $\rho_{2}(n, \theta)$ for $\varepsilon=\varepsilon_{0}$, the proof is completed.

Corollary 3. Assume that $\theta=1$ and that $f_{2}$ satisfies (37) with $(n+2) /(n)$ $<\rho_{2} \leqslant(n+4) /(n)$. Let $\mathcal{F}_{2}$ be the map defined by

$$
\mathcal{F}_{2}\left(\left[\begin{array}{l}
u \\
v
\end{array}\right]\right)=\left[\begin{array}{c}
0 \\
F_{2}(v)
\end{array}\right]
$$

where $F_{2}(v)$ is the Nemitskil map associated with $f_{2}$. Then, $\mathcal{F}_{2}$ is an $\varepsilon$-regular map relative to $\left(Y_{(\theta)_{-1}}^{1}, Y_{(\theta)_{-1}}\right)$ for $\varepsilon \in\left[\left(n \rho_{2}-n-2\right) /\left(4 \rho_{2}\right), 1 /\left(2 \rho_{2}\right)\right]$ and $\gamma(\varepsilon) \in\left[\rho_{2} \varepsilon,(1 / 2)\right]$. That is,

$$
\begin{aligned}
& \left\|\mathcal{F}_{2}\left(\left[\begin{array}{l}
u \\
v
\end{array}\right]\right)-\mathcal{F}_{2}\left(\left[\begin{array}{l}
u^{\prime} \\
v^{\prime}
\end{array}\right]\right)\right\|_{Y_{(\theta)-1}^{\gamma(\varepsilon)}} \\
& \leqslant c\left\|\left[\begin{array}{l}
u \\
v
\end{array}\right]-\left[\begin{array}{c}
u^{\prime} \\
v^{\prime}
\end{array}\right]\right\|_{Y_{(\theta)-1}^{1+\varepsilon}}\left(1+\left\|\left[\begin{array}{c}
u \\
v
\end{array}\right]\right\|_{Y_{(\theta)-1}^{1+\varepsilon}}^{\rho_{2}-1}+\left\|\left[\begin{array}{c}
u^{\prime} \\
v^{\prime}
\end{array}\right]\right\|_{Y_{(\theta)_{-1}}^{1+\varepsilon}}^{p_{2}-1}\right),\left[\begin{array}{l}
u \\
v
\end{array}\right],\left[\begin{array}{c}
u^{\prime} \\
v^{\prime}
\end{array}\right] \in Y_{(\theta)-1}^{1+\varepsilon},
\end{aligned}
$$

whenever $\varepsilon \in\left[\left(n \rho_{2}-n-2\right) /\left(4 \rho_{2}\right), 1 /\left(2 \rho_{2}\right)\right], \gamma(\varepsilon) \in\left[\rho_{2} \varepsilon,(1 / 2)\right]$.

The intervals of $\varepsilon$-regularity of $\mathcal{F}_{2}$ are a function of the growth exponent $\rho_{2}$. In Figure 2 the reader can see how in the case $\theta=1$ the intervals of $\varepsilon$-regularity change as a function of $\rho_{2}$.

COROLLARY 4. Let $\mathcal{F}$ in (19) be given by

$$
\mathcal{F}\left(\left[\begin{array}{l}
u \\
v
\end{array}\right]\right)=\mathcal{F}_{1}\left(\left[\begin{array}{l}
u \\
v
\end{array}\right]\right)+\mathcal{F}_{2}\left(\left[\begin{array}{l}
u \\
v
\end{array}\right]\right),
$$

with $\mathcal{F}_{1}, \mathcal{F}_{2}$ being Nemitskin maps associated with $f_{1}, f_{2}$ respectively. Suppose that $f_{1}$ is restricted by (32) and (33), and $f_{2}$ is restricted by (37) and (38). Then,

1. $\mathcal{F}_{1}$ satisfies condition $(36)$ with parameters $\varepsilon=(n-2) /(2(n+2))=: \varepsilon_{1}$, $\gamma(\varepsilon)=1 / 2=: \gamma_{1}\left(\varepsilon_{1}\right)$,

2. $\mathcal{F}_{2}$ satisfies condition (43) with parameters $\varepsilon=n /(2 n+8 \theta)=: \varepsilon_{2}, \gamma(\varepsilon)$ $=1 / 2=: \gamma_{2}\left(\varepsilon_{2}\right)$, 


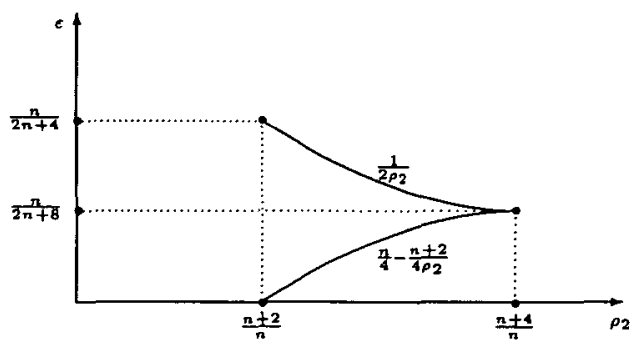

Figure 2: Intervals of $\varepsilon$-regularity for $\mathcal{F}_{2}$ and $\theta=1$

where $\theta \in[1 / 2,1]$ and $\varepsilon_{1}, \varepsilon_{2}, \gamma_{1}\left(\varepsilon_{1}\right), \gamma_{2}\left(\varepsilon_{2}\right)$ are such that

$$
\min \left\{\gamma_{i}\left(\varepsilon_{i}\right) ; i=1,2\right\}=1 / 2=\tilde{\gamma}>\tilde{\varepsilon}=\frac{n}{2 n+8 \theta}=\max \left\{\varepsilon_{i} ; i=1,2\right\} .
$$

In particular, $\mathcal{F}$ in (44) fulfills the assumptions required for nonlinearity in Proposition 6.

We are now ready to formulate the following existence theorem.

THEOREM 3. Consider (19) as a counterpart of (1) in the case when $f(u, v)$ $=f_{1}(u)+f_{2}(v)$ and functions $f_{i}: \mathbb{R} \rightarrow \mathbb{R}, i=1,2$ are as in Corollary 4. Let $\left[\begin{array}{l}\widetilde{u}_{0} \\ \tilde{v}_{0}\end{array}\right] \in Y^{0}$ and $B_{Y^{0}}\left(\left[\begin{array}{c}\widetilde{u}_{0} \\ \widetilde{v}_{0}\end{array}\right], r\right)$ be a ball in $Y^{0}$ with radius $r>0$ centred at $\left[\begin{array}{l}\tilde{u}_{0} \\ \widetilde{v}_{0}\end{array}\right]$.

Then, there are $r>0$ and $\tau_{0}>0$ such that for each $\left[\begin{array}{c}u_{0} \\ v_{0}\end{array}\right] \in B_{Y^{0}}\left(\left[\begin{array}{c}\tilde{u}_{0} \\ \tilde{v}_{0}\end{array}\right], r\right)$ there exists a unique $\widetilde{\varepsilon}$-regular solution $\left[\begin{array}{l}u \\ v\end{array}\right]\left(\cdot, u_{0}, v_{0}\right)$ to (19). In addition,

(i) $t^{\zeta}\left\|\left[\begin{array}{l}u \\ v\end{array}\right]\left(t, u_{0}, v_{0}\right)\right\|_{Y_{(\theta)_{-1}}^{1+\zeta}} \rightarrow 0$ as $t \rightarrow 0^{+}, 0<\zeta<1 / 2$,

(ii) $t^{\zeta}\left\|\left[\begin{array}{l}u \\ v\end{array}\right]\left(t, u_{1}, v_{1}\right)-\left[\begin{array}{l}u \\ v\end{array}\right]\left(t, u_{2}, v_{2}\right)\right\|_{Y_{(\theta)-1}^{1+\zeta}} \leqslant C^{\prime}\left\|\left[\begin{array}{l}u_{1} \\ v_{1}\end{array}\right]-\left[\begin{array}{l}u_{2} \\ v_{2}\end{array}\right]\right\|_{Y^{0}}$ whenever $t \in\left[0, \tau_{0}\right], 0 \leqslant \zeta \leqslant \zeta_{0}<1 / 2,\left[\begin{array}{l}u_{1} \\ v_{1}\end{array}\right],\left[\begin{array}{l}u_{2} \\ v_{2}\end{array}\right] \in B_{Y^{0}}\left(\left[\begin{array}{l}\tilde{u}_{0} \\ \tilde{v}_{0}\end{array}\right], r\right)$,

(iii) $\left[\begin{array}{l}u \\ v\end{array}\right]\left(\cdot, u_{0}, v_{0}\right) \in C\left(\left(0, \tau_{0}\right], Y_{(\theta)-1}^{1+(1 / 2)}\right) \cap C^{1}\left(\left(0, \tau_{0}\right], Y_{(\theta)-1}^{1+\zeta}\right)$ for $0 \leqslant \zeta<1 / 2$; in particular the solution, $\left[\begin{array}{l}u \\ v\end{array}\right]\left(\cdot, u_{0}, v_{0}\right)$ satisfies both relations in (19). 
Proof: The assertion follows from Corollary 4 and Proposition 6 .

3.3. Further comments on local existence. Proof of Theorem 1. Using the original results of $[2,3]$ concerning the existence of $\varepsilon$-regular solutions (see Subsection 3.1), one cannot take advantage of working with coordinates. In this subsection we consider an extension of these results to include systems with different $\varepsilon$-regular properties in each coordinate.

More specifically we consider problems of the form (19) where $\mathcal{A}_{(\theta)_{-1}}$ is the sectorial operator previously defined in $Y_{(\theta)_{-1}}$ with domain $Y^{0}=Y_{(\theta)_{-1}}^{1}=X^{1 / 2} \times X$.

Assume that there are constants $\varepsilon_{1}>0, \varepsilon_{2}>0, \rho_{1}>1, \rho_{2}>1, \gamma_{1}\left(\varepsilon_{1}\right) \in\left[\rho_{1} \varepsilon_{1}, 1\right)$, $\gamma_{2}\left(\varepsilon_{2}\right) \in\left(\rho_{2} \varepsilon_{2}, 1\right)$ such that $\mathcal{F}\left(\left[\begin{array}{l}u \\ v\end{array}\right]\right)=\left[\begin{array}{c}0 \\ F(u, v)\end{array}\right]$ satisfies

$$
\begin{array}{ll}
\| F(u, v) & -F\left(u^{\prime}, v\right) \|_{X^{-(1 / 2)+\gamma_{1}\left(\varepsilon_{1}\right)(1-\theta)}} \\
& \leqslant c_{1}\left\|u-u^{\prime}\right\|_{X^{\left(1+2 \varepsilon_{1}(1-\theta)\right) / 2}}\left(1+\|u\|_{X^{\left(1+2 \varepsilon_{1}(1-\theta)\right) / 2}}^{\rho_{1}-1}+\left\|u^{\prime}\right\|_{X^{\left(1+2 \varepsilon_{1}(1-\theta)\right) / 2}}^{\rho_{1}-1},\right. \\
& u, u^{\prime} \in X^{\left(1+2 \varepsilon_{1}(1-\theta)\right) / 2}, \\
\| F(u, v)- & F\left(u, v^{\prime}\right) \|_{X^{-(1 / 2)+\gamma_{2}\left(\varepsilon_{2}\right)(1-\theta)}} \\
& \leqslant c_{2}\left\|v-v^{\prime}\right\|_{X^{\theta \varepsilon_{2}}}\left(1+\|v\|_{X^{\theta \varepsilon_{2}}}^{\rho_{2}-1}+\left\|v^{\prime}\right\|_{X^{\theta \varepsilon_{2}}}^{\rho_{2}-1}\right), v, v^{\prime} \in X^{\theta \varepsilon_{2}} .
\end{array}
$$

Under these conditions we have the following extension of the results in $[2,3]$.

Proposition 7. Let $\widetilde{y}_{0} \in Y^{0}$ and $B_{Y^{0}}\left(\tilde{y}_{0}, r\right)$ denote a ball in $Y^{0}$ with radius $r>0$ centred at $\widetilde{y}_{0}$. Suppose also that

$$
\min \left\{\gamma_{i} ; i=1,2\right\}=: \widetilde{\gamma}>\widetilde{\varepsilon}:=\max \left\{\varepsilon_{i} ; i=1,2\right\} .
$$

Then, there are $r>0$ and $\tau_{0}>0$ such that for each $y_{0} \in B_{Y^{0}}\left(\widetilde{y}_{0}, r\right)$ there exists a unique $\tilde{\varepsilon}$-regular solution $y=y\left(\cdot, y_{0}\right)$ to (19). In addition,

(i) $t^{\zeta}\left\|y\left(t, y_{0}\right)\right\|_{Y_{(\theta)-1}^{1+\zeta}} \rightarrow 0$ as $t \rightarrow 0^{+}, 0<\zeta<\tilde{\gamma}$,

(ii) $t^{\zeta}\left\|y\left(t, y_{1}\right)-y\left(t, y_{2}\right)\right\|_{Y_{(\theta)-1}^{1+\zeta}} \leqslant C^{\prime}\left\|y_{1}-y_{2}\right\|_{Y^{0}}, t \in\left[0, \tau_{0}\right]$, for $0 \leqslant \zeta \leqslant \zeta_{0}<\tilde{\gamma}$, $y_{1}, y_{2} \in B_{Y^{0}}\left(\widetilde{y}_{0}, r\right)$

(iii) $y \in C\left(\left(0, \tau_{0}\right], Y_{(\theta)-1}^{1+\tilde{\gamma}}\right) \cap C^{1}\left(\left(0, \tau_{0}\right], Y_{(\theta)-1}^{1+\zeta}\right), \zeta<\tilde{\gamma}$, and, in particular, $y$ satisfies both relations in (19).

The hypothesis on the map $F$ allows us to consider maps that may not be decomposed into a sum of maps each of them depending only on one coordinate.

The proof of the above result follows step by step the proof of a similar result contained in $[2,3]$. We must only be careful to see that when we need an estimate on the norm of $F(u, v)$ we must decompose it into $F=F_{1}+F_{2}$ where $F_{1}(u, v)=F(u, 0)$ 
and $F_{2}(u, v)=F(u, v)-F(u, 0)$. Also note that, from (46) and (47) we have

$$
\begin{aligned}
\left\|F_{1}(u, v)\right\|_{X^{-(1 / 2)+\gamma_{1}\left(e_{1}\right)(1-\theta)}} & \leqslant F(0,0) \|_{X^{-(1 / 2)+\gamma_{1}\left(e_{1}\right)(1-\theta)}} \\
& +c_{1}\|u\|_{X^{\left(1+2 \varepsilon_{1}(1-\theta)\right) / 2}}\left(1+\|u\|_{X^{\left(1+2 \varepsilon_{1}(1-\theta)\right) / 2}}^{\rho_{1}-1}\right) \\
& \leqslant \widetilde{c}_{1}\left(1+\|u\|_{X^{\left(1+2 \varepsilon_{1}(1-\theta)\right) / 2}}^{\rho_{1}}\right)
\end{aligned}
$$

and

$$
\begin{aligned}
\left\|F_{2}(u, v)\right\|_{X^{-(1 / 2)+\gamma_{2}\left(\varepsilon_{2}\right)(1-\theta)}} & =\|F(u, v)-F(u, 0)\|_{X^{-(1 / 2)+\gamma_{2}\left(\varepsilon_{2}\right)(1-\theta)}} \\
& \leqslant c_{2}\|v\|_{X^{\theta \varepsilon_{2}}}\left(1+\|v\|_{X^{\theta \varepsilon_{2}}}^{\rho_{2}-1}\right) \\
& \leqslant \widetilde{c}_{2}\left(1+\|v\|_{X^{\theta \varepsilon_{2}}}^{\rho_{2}}\right) .
\end{aligned}
$$

On the other hand, when we wish to estimate $F(u, v)-F\left(u^{\prime}, v^{\prime}\right)$ we must decompose it into the sum

$$
F(u, v)-F\left(u^{\prime}, v^{\prime}\right)=\left[F(u, v)-F\left(u^{\prime}, v\right)\right]+\left[F\left(u^{\prime}, v\right)-F\left(u^{\prime}, v^{\prime}\right)\right]
$$

and use the assumption (46).

In the application to the strongly damped wave equation (1) we observe that the map $\mathcal{F}$ is given by

$$
\mathcal{F}\left(\left[\begin{array}{l}
u \\
v
\end{array}\right]\right)=\left[\begin{array}{c}
0 \\
F(u, v)
\end{array}\right]
$$

where $F(u, v)$ is the Nemitskiu map originated from a function $f: \mathbb{R}^{2} \rightarrow \mathbb{R}$. Assume that the function $f: \mathbb{R}^{2} \rightarrow \mathbb{R}$ satisfies (7) with $1<\rho_{1} \leqslant(n+2) /(n-2)$ and $1<\rho_{2}$ $\leqslant(n+4 \theta) / n$. Then the inequalities in (46) are satisfied with parameters $\varepsilon_{i}, \gamma_{i}\left(\varepsilon_{i}\right)$, $i=1,2$ such that (48) holds. Proposition 7 can be then applied to obtain the local well posedness of (1). Theorem 1 is thus proved.

\section{REFERENCES}

[1] H. Amann, Linear and quasilinear parabolic problems (Birkhäuser, Basel, 1995).

[2] J.M. Arietta and A.N. Carvalho, 'Abstract parabolic problems with critical nonlinearities and applications to Navier-Stokes and heat equations', Trans. Amer. Math. Soc. 352 (2000), 285-310.

[3] J.M. Arietta, A.N. Carvalho and A. Rodriguez-Bernal, 'Parabolic problems with nonlinear boundary conditions and critical nonlinearities', J. Differential Equations 156 (1999), 376-406.

[4] S. Chen and R. Triggiani, 'Proof of two conjectures of G. Chen and D.L. Russell on structural damping for elastic systems', in Lecture Notes in Mathematics 1354 (Springer-Verlag, 1988), pp. 234-256.

[5] S. Chen and R. Triggiani, 'Proof of extension of two conjectures on structural damping for elastic systems: The case $\frac{1}{2} \leqslant \alpha \leqslant 1$ ', Pacific J. Math. 136 (1989), 15-55. 
[6] S. Chen and R. Triggiani, 'Characterization of domains of fractional powers of certain operators arising in elastic systems, and applications', J. Differential Equations 88 (1990), 279-293.

[7] H. Fujita and T. Kato, 'On the Navier-Stokes initial value problem. I', Arch. Rational Mech. Anal. 16 (1964), 269-315.

[8] T. Kato, 'Fractional powers of dissipative operators II', J. Math. Soc. Japan 14 (1962), 242-248.

[9] H. Triebel, Interpolation theory, function spaces, differential operators (Veb Deutscher, Berlin, 1978).

Departamento de Matemática

Instituto de Ciências Matemáticas e de Computação

Universidade de São Paulo

- Campus de São Carlos

Caixa Postal 668

13.560-970 São Carlos SP,

Brazil

e-mail: andcarva@icmc.sc.usp.br
Institute of Mathematics

Silesian University

40-007 Katowice

Poland

e-mail: jcholewa@ux2.math.us.edu.pl 